\title{
Comparative Study of the Effect of the Parameters of Sizing Data on Results by the Meshless Methods (MLPG)
}

\author{
Ahmed Moussaoui, Touria Bouziane \\ Team Mechanics and Energy System Lab LAMP, Faculty of Sciences, Moulay Ismail University, Meknes, Morocco \\ Email: moussaoui.physique@gmail.com
}

Received November 29, 2012; revised December 30, 2012; accepted January 10, 2013

\begin{abstract}
The local Petrov-Galerkin methods (MLPG) have attracted much attention due to their great flexibility in dealing with numerical model in elasticity problems. It is derived from the local weak form (WF) of the equilibrium equations and by inducting the moving last square approach for trial and test functions in (WF) is discussed over local sub-domain. In this paper, we studied the effect of the configuration parameters of the size of the support or quadrature domain, and the effect of the size of the cells with nodes distribution number on the accuracy of the methods. It also presents a comparison of the results for the Shear stress, the deflections and the error in energy.
\end{abstract}

Keywords: MLPG; Meshless Method; Linear Elasticity; Cantilever Plates; Support Domain

\section{Introduction}

Recently Meshless formulations are becoming popular due to their higher adaptivity and lower cost for preparing input data in the numerical analysis. A variety of meshless methods has been proposed so far (Belytschko et al., 1994; Atluri and Shen, 2002; Liu, 2003; Atluri, 2004) [1-6]. Many of them are derived from a weak-form formulation on global domain [1] or a set of local subdomains [4-7].

The meshless local Petrov-Galerkin (MLPG) method originated by Atluri and Zhu [1] uses the so-called local weak form of the Petrov-Galerkin formulation. MLPG has been fine-tuned, improved, and extended by Atluri's group (Atluri et al., 1999) and other researchers over the years [8-10]. MLPG has been applied to solve elastostatics and elastodynamics problems of solids and plats [11].

The method is a fundamental base for the derivation of many meshless formulations, since trial and test functions are chosen from different functional spaces.

MLPG does not need a global mesh for either function approximation or integration. The procedure is quite similar to numerical methods based on the strong-form formulation, such as the finite difference method (FDM). However, because in the MLPG implementation, moving least squares (MLS) approximation is employed for constructing shape functions, special treatments are needed to enforce the essential boundary conditions $[4,7]$.

The aims of this paper are to study the effect on accuracy and convergence of MLPG methods of different size parameters: $\alpha_{s}$ and $\alpha_{Q}$ associated to support and qua- drature domains respectively. The support domain is denoted be equal to influence domain. For fixed values of: $\alpha_{s}$ and $\alpha_{Q}$, the effect of cells numbers $n_{c}$ with nodes distribution number, on energy errors is also studied and some of our results are presented.

In this work, the MLPG method will be developed for solving the problem of a thin elastic homogenous plate. The discretization and numerical implementation are presented in Section 2 numerical example for 2D problem are given in Section 3. Then paper ends with discussions and conclusions.

\section{Basic Equations}

Let us consider a two-dimensional problem of solid mechanics in domain $\Omega$ bounded by $\Gamma$ whose strongform of governing equation and the essential boundary conditions are given by:

$$
\begin{gathered}
\sigma_{i j, j}(x)+b_{i}(x)=0 \\
\sigma_{i j} n_{j}=\bar{t}_{i} \text { on } \Gamma_{t} \\
u_{i}=\bar{u}_{i} \text { on } \Gamma_{u}
\end{gathered}
$$

where in $\Omega, \sigma^{\mathrm{T}}=\left[\sigma_{x x}, \sigma_{y y}, \tau_{x y}\right]$ is the stress vector and $b^{\mathrm{T}}=\left[b_{x}, b_{y}\right]$ the body force vector.

On the natural boundaries $\bar{t}$ is the prescribed traction, $\boldsymbol{n}=\left(n_{1}, n_{2}\right)$ denoted the vector of unit outward normal at a point.

$\left[u_{1}, u_{2}\right]$ the displacement components in the plan and $\left[\bar{u}_{1}, \bar{u}_{2}\right]$ on the essential boundaries.

In the local Petrov-Galerkin approaches [3], one may 
write a weak form over $\Omega_{Q}$ a local quadrature domain (for node I), which may have an arbitrary shape, and contain the point $x_{Q}$ in question, (see Figure 1). The generalized local weak form of the differential Equations (1) and (3) is obtained by:

$$
\begin{aligned}
& \int_{\Omega_{Q}}\left(\sigma_{i j, j}(x)+b_{i}(x)\right) W_{I} \mathrm{~d} \Omega \\
& -\alpha \int_{\Gamma_{Q u}}\left(u_{i}-\bar{u}_{i}\right) W_{I} \mathrm{~d} \Gamma=0
\end{aligned}
$$

where $\Omega_{Q}$ is the local domain of quadrature for node I and $\Gamma_{Q u}$ is the part of the essential boundary that intersect with the quadrature domain $\Omega_{O}$. $W_{I}$ is the weight or test function, $W_{I} \in C^{K}(\Omega)$ [12]. The first term in Equation (4) is for the equilibrium (in locally weighted average sense) requirement at node I. The second integral in Equation (4) is the curve integral to enforce the essential boundary conditions, because the MLS shape functions used in MLPG lack the Kronecker delta function property.

$\alpha$ is the penalty factor, Here we use the same penalty factor for all the displacement constraint equations (essential boundary conditions) [1]

Generally, in meshfree methods, the representation of field nodes in the domain will be associated to other repartitions of problem domain: influence domain for nodes interpolation, $\Omega_{S}$ is the support domain for accuracy. For each node $\Omega_{W}$ is the weight function domain, and $\Omega_{Q}$ is the quadrature domain for local integration.

Using the divergence theorem [11] in Equation (4) we obtain:

$$
\begin{aligned}
& \int_{\Gamma_{Q}} \sigma_{i j} n_{j} W_{I} \mathrm{~d} \Gamma-\int_{\Omega_{Q}} \sigma_{i j} W_{I, j} \mathrm{~d} \Omega \\
& +\int_{\Omega_{Q}} b_{i} W_{I} \mathrm{~d} \Omega-\alpha \int_{\Gamma_{Q u}}\left(u_{i}-\bar{u}_{i}\right) W_{I} \mathrm{~d} \Gamma=0
\end{aligned}
$$

where $\Gamma_{Q}=\Gamma_{Q 0} \cup \Gamma_{Q u} \cup \Gamma_{Q t}$

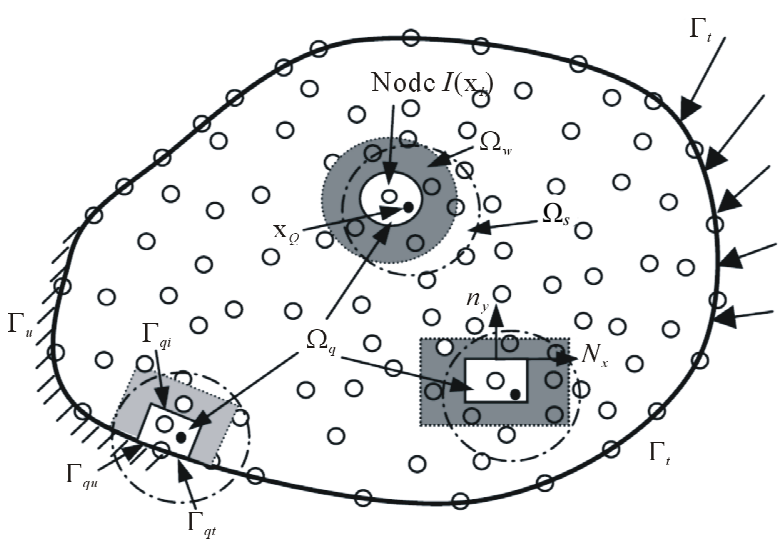

Figure 1. The local sub-domains around point $x_{Q}$ and boundaries.
$\Gamma_{Q 0}:$ The internal boundary of the quadrature domain

$\Gamma_{Q t}$ : The part of the natural boundary that intersects with the quadrature domain

$\Gamma_{Q u}$ : The part of the essential boundary that intersects with the quadrature domain

When the quadrature domain $\Omega_{Q}$ is located entirely within the global domain on $\Gamma_{Q t}$ and $\Gamma_{Q u}$ no boundary conditions are specified then $\Gamma_{Q}=\Gamma_{Q 0}$.

Unlike the Galerkin method, the Petrov-Galerkin method chooses the trial and test functions from different spaces. The weight function $W_{I}$ is purposely selected in such a way that it vanishes on $\Gamma_{Q 0}$. We can then change the expression of Equation (5):

$$
\begin{aligned}
& \int_{\Omega_{Q}} \sigma_{i j} W_{I, j} \mathrm{~d} \Omega+\alpha \int_{\Gamma_{Q u}} u_{i} W_{I} \mathrm{~d} \Gamma-\int_{\Gamma_{Q u}} \sigma_{i j} n_{j} W_{I} \mathrm{~d} \Gamma \\
& =\int_{\Gamma_{Q t}} \bar{t}_{i} W_{I} \mathrm{~d} \Gamma+\alpha \int_{\Gamma_{Q u}} \bar{u}_{i} W_{I} \mathrm{~d} \Gamma+\int_{\Omega_{Q}} b_{i} W_{I} \mathrm{~d} \Omega
\end{aligned}
$$

Witch is the local Petrov-Galerkin weak form. Here we require $u_{i} \in C^{0}\left(\Omega_{Q}\right)[3,11]$ and the simplified Petrov-Galerkin form is:

$$
\int_{\Omega_{Q}} \sigma_{i j} W_{I, j} \mathrm{~d} \Omega=\int_{\Omega_{Q}} b_{i} W_{I} \mathrm{~d} \Omega
$$

Precedent equations are used to establish the discrete equations for all the nodes whose quadrature domain falls entirely within the problem domain (Equation (7)) and to establish the discrete equations for all the boundary nodes or the nodes whose quadrature domain intersects with the problem boundary "Equation (6)".

To approximate the distribution of the function $u$ in $\Omega_{S}$ the support domain over a number of nodes $n_{0}$. We shall have the approximant $u^{h}(x)$ of $u$ [13]

$$
\forall x \in \Omega_{S}: u^{h}(x)=\sum_{I=1}^{n_{0}} \Phi_{I}(x) u_{I}
$$

where $I$ denote the set of the nodes in the support domain $\Omega_{S}$ of point $x_{Q}$.

$\Phi_{I}$ the MLS shape function for node I that is created using nodes in the support domain $\Omega_{S}$ of point $x_{Q}$. The discrete system in Equation (6) is given in matrix form:

$$
\begin{aligned}
& \int_{\Omega_{Q}} V_{I}^{T} \sigma \mathrm{d} \Omega+\alpha \int_{\Gamma_{Q u}} \bar{u} W_{I} \mathrm{~d} \Gamma-\int_{\Gamma_{Q u}} t W_{I} \mathrm{~d} \Gamma \\
& =\int_{\Gamma_{Q t}} \bar{t} W_{I} \mathrm{~d} \Gamma+\alpha \int_{\Gamma_{Q u}} \bar{u} W_{I} \mathrm{~d} \Gamma+\int_{\Omega_{Q}} W_{I} b \mathrm{~d} \Omega
\end{aligned}
$$

where $V_{I}=\left(\begin{array}{cc}W_{I, x} & 0 \\ 0 & W_{I, y} \\ W_{I, y} & W_{I, x}\end{array}\right)$ is a matrix that collects the derivatives of the weight functions in Equation (6), and $W=\left(\begin{array}{cc}W_{I} & 0 \\ 0 & W_{I}\end{array}\right)$ is the matrix of weight function. The stress vector defined by: 


$$
\sigma=C \varepsilon=C L_{d} u^{h}
$$

where $C$ is the symmetric elasticity tensor of the material

$$
C=\left(\begin{array}{ccc}
E / 1-v^{2} & v E / 1-v^{2} & 0 \\
v E / 1-v^{2} & E / 1-v^{2} & 0 \\
0 & 0 & E / 2(1+v)
\end{array}\right)
$$

Substituting the differential operator

$$
L_{d}=\left(\begin{array}{cc}
\partial / \partial x & 0 \\
0 & \partial / \partial y \\
\partial / \partial y & \partial / \partial x
\end{array}\right)
$$

and Equation (8) into Equation (10) we obtain:

$$
\sigma=C \sum_{I=1}^{n_{0}} B_{I} u_{I}
$$

where $B_{I}=\left(\begin{array}{cc}\Phi_{I, x} & 0 \\ 0 & \Phi_{I, y} \\ \Phi_{I, y} & \Phi_{I, x}\end{array}\right)$ and by using $L_{n}=\left(\begin{array}{cc}n_{1} & 0 \\ 0 & n_{2} \\ n_{2} & n_{1}\end{array}\right)$ the tractions of a point $x$ can be written as:

$$
t=L_{n}^{T} \sigma
$$

Substituting Equations (8), (11) and (12) into Equation (6), we obtain the discrete systems of linear equations for the node $I$.

$$
\begin{aligned}
& \sum_{I=1}^{n_{0}}\left[\int_{\Omega_{Q}} V_{I}^{T} C B_{I} \mathrm{~d} \Omega+\alpha \int_{\Gamma_{Q u}} W_{I} \Phi_{I} \mathrm{~d} \Gamma\right. \\
& \left.\quad-\int_{\Gamma_{Q}} W_{I} L_{n}^{T} C B_{I} \mathrm{~d} \Gamma\right] u_{I} \\
& =\int_{\Gamma_{Q t}} \bar{t} W_{I} \mathrm{~d} \Gamma+\alpha \int_{\Gamma_{Q u}} \bar{u} W_{I} \mathrm{~d} \Gamma+\int_{\Omega_{Q}} W_{I} b \mathrm{~d} \Omega
\end{aligned}
$$

That can assembled in matrix form:

$$
\sum_{I=1}^{n_{0}} K_{I} u_{I}=f_{I}
$$

where nodal stiffness matrix

$$
\begin{aligned}
K_{I} & =\int_{\Omega_{Q}} V_{I}^{T} C B_{I} \mathrm{~d} \Omega+\alpha \int_{\Gamma_{Q u}} W_{I} \Phi_{I} \mathrm{~d} \Gamma \\
& -\int_{\Gamma_{Q}} W_{I} L_{n}^{T} C B_{I} \mathrm{~d} \Gamma
\end{aligned}
$$

And nodal force vector with contributions from body forces applied in the problem domain, tractions applied on the natural boundary, as well as the penalty force terms.

$$
f_{I}=\int_{\Gamma_{Q t}} \bar{t} W_{I} \mathrm{~d} \Gamma+\alpha \int_{\Gamma_{Q u}} \bar{u} W_{I} \mathrm{~d} \Gamma+\int_{\Omega_{Q}} W_{I} b \mathrm{~d} \Omega
$$

Two independent linear equations can be obtained for each node in the entire problem domain and assembled all these $2 * n$ equations to obtain the final global sys- tem equations:

$$
K_{2 n * 2 n} U_{2 n * 1}=F_{2 n * 1}
$$

To solve the precedent system, the standard Gauss quadrature formula is applied with 16 Gauss points $[3,14]$ for evaluation of boundary and domain integrals in Equations (15) and (16)

\section{Numerical Example}

In this section, numerical results are presented for Cantilever rectangular plate in Figure 2. First we investigate the effects of the size of support or quadrature domains and we examine the numerically convergence of MLPG, then comparisons will be made with the analytic solution [15]

The problem data:

The height of the beam $D=12 \mathrm{~m}$ and the length of the beam: $L=48 \mathrm{~m}$;

The thickness of the plat: unit and Loading (integration of the distributed traction): $P=10^{3} \mathrm{~N}$;

Young's modulus: $E=3 \times 10^{7} \mathrm{~N} / \mathrm{m}^{2}$ and Poisson's ratio: $v=0.3$.

The standard Gaussian quadrature formula is applied with 16 Gauss points, and for MLS approximation linear polynomial basis functions are applied, the cubic spline function is used as the test function for the local Petrov-

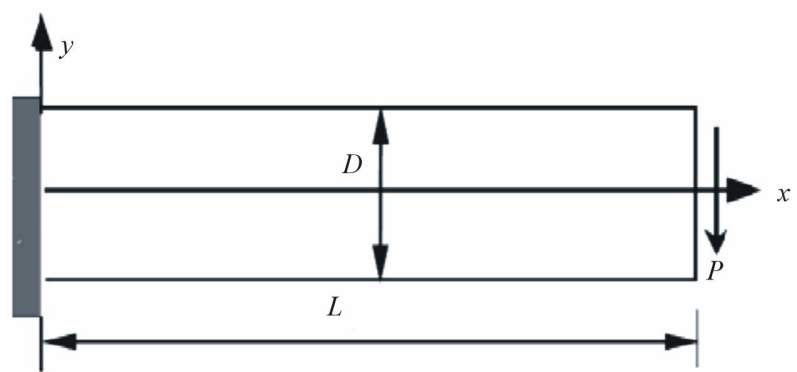

Figure 2. Cantilever plate subjected to distributed traction at the free end.

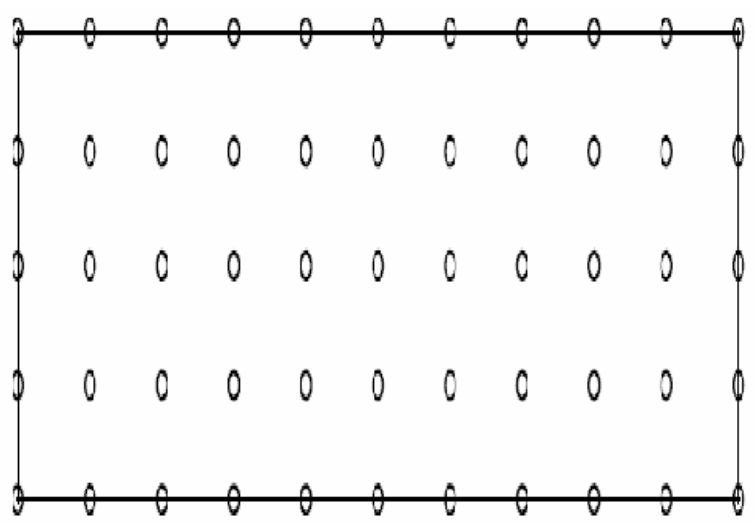

Figure 3. 55 regular field nodes on the problem domain and boundaries. 
Galerkin weak-form. In our numerical calculations we consider many regular distributions of nodes: 55 or 175 . To calculate the error energy a background cells is required, then we have varying the number of cell. To obtain the distribution of the deflection and stress through the plates, size of quadrature domain and support domain are varied. Nodal configuration for a cantilever plate with 55 nodes (Figure 3) (nodal distance $d_{c x}=4.8, d_{c y}=3$ ) and the sizes of $\Omega_{Q}$ is defined by: $r_{Q}=\alpha_{Q} d_{c I}$ where $d_{c I}$ is the nodal spacing near node $I$ and $r_{Q}$ is the size of the local quadrature domain for node $I$. The sizes of quadrature domains will be, there fore determined by $\Omega_{Q x}$ and $\alpha_{Q y}$ which are dimensionless coefficients in $x$ and $y$ directions, respectively. For simplicity

$\alpha_{Q x}=\alpha_{Q y}=\alpha_{Q}$ is used. The dimension of the support domain is determined by $d_{s}=\alpha_{s} d_{c}$ and $\alpha_{s}$ is the dimensionless size of support domain.

\section{Discussions}

Figure 4 Shows the variation of the effective transverse shear stress $\tau_{x y}$ at different points on vertical of the plate by varying $x$ for $\alpha_{s}=3$ and $\alpha_{Q}=1.5$. It can be seen the shear stress distributions on the cross-section at in other sections $(x=L / 4, x=L / 2, x=3 L / 4$ and $x=L)$. it's shown that the shape is identical to that obtained by theoretical analysis ( section $x=L$ ).

The accuracy is clear for the greater value of field nodes distribution. It is also shown in this figure, on the cross-section the meshless MLPG agree well with those from analytical solution (dashed lines).

Figure 5 displays the variation of the energy error as a function of the size of the local support domain, for fixed value of $\alpha_{Q}$, a background cells is needed, we take $\alpha_{Q}=1.5$. We note on the figure the effect distribution field nodes number on the result, we take $n=55$ and 175 number of cell is $n_{c}=40$ and 144 respectively.

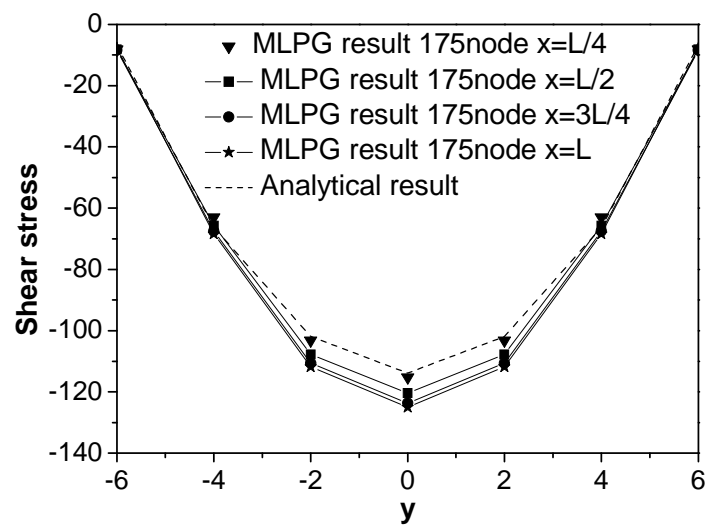

Figure 4. Shear stress $\left(\tau_{x y}\right)$ distribution as a function of $y$ for different values of $x(x=L / 4, x=L / 2, x=3 L / 4$ and $x=L$ ) ( 175 regular field nodes).
In the case $\alpha_{Q}=1.5$ For the value of field nodes number $n=55$ (number of cell is $n_{c}=40$ ) the result gives a greater domain when the value of $\alpha_{s}: 1.85 \leq \alpha_{s} \leq 5$ can be selected and the method is yet convergent.

For $n=175\left(\alpha_{Q}=1.5\right)$ the domain when accuracy is good in $1.8 \leq \alpha_{s} \leq 3$ our results are comparable to those obtained by other authors $[3,5,6]$.

But in case $\alpha_{Q}=2$, we have considered $n=175$ the domain of convergence is found to be greater $1.85 \leq \alpha_{s} \leq 3.65$.

Figure 6 displays the variation of the energy error as a function of the size of the local quadrature domain, for fixed value of $\alpha_{s}$ a background cells used, we take $\alpha_{s}=3$ and we choose two values of $n=55$ and 175 number of cell is $n_{c}=40$ and 144 respectively.

For the selected values $n$ the domain when the value of $\alpha_{Q}$ can be selected and the method is yet convergent is $\left(1.5 \leq \alpha_{Q} \leq 3\right)$.

In Figure 7 the deflection results are plotted as function of $x$ and fixed value of $\alpha_{Q}=1.5$ where $y=0$ and by varying the size of the support domain $\left(\alpha_{s}=2 ; 2.5 ; 3 ; 3.5\right)$. It can be seen that the function presents a classical shape

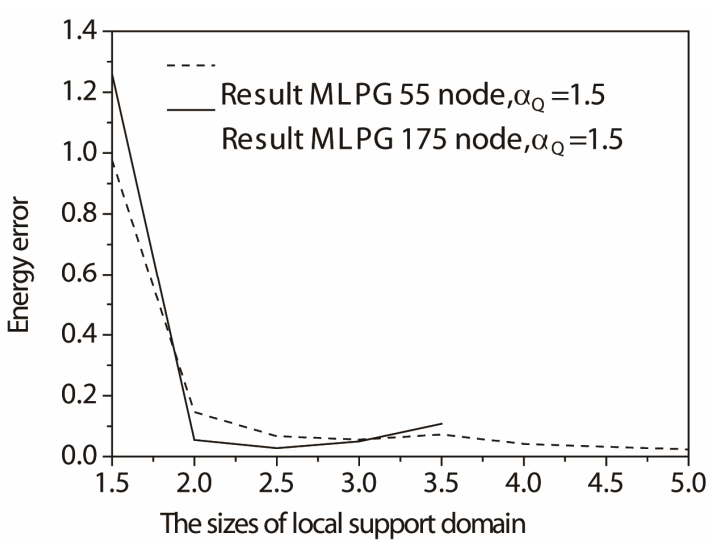

Figure 5. Influence of the $\alpha_{s}$ on energy error for different distribution nodes numbers.

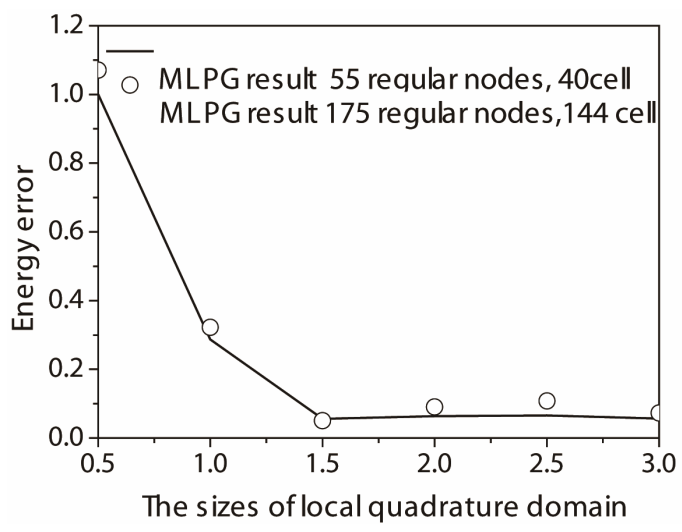

Figure 6. Influence of the $\alpha_{Q}$ on energy-error for two distribution field nodes numbers. 
for all values of $\alpha_{s}$ and reaches the analytical solution.

The effect of the field distribution number on the deflection values is presented in Figure 8. For different values of $\alpha_{s}$ and $\alpha_{Q}$ A Comparison is made with the analytical results of deflection (solid line plotted in the Figure 8).

For $\alpha_{Q}=2$ the greater values of nodes numbers $n=$ 175 even gives precision for the greater values of $\alpha_{s}=3.65$ (dashed curve). No curve is available for $n=$ 55.

For the less values of $\alpha_{s}$-we have found in Figure 5 $\alpha_{s}=1.85$ - two curves are plotted for $n=55$ and $n=$ 175.

It also presented the variation of the defection in the case $\alpha_{s}=3.65, \alpha_{Q}=2$ for $n=55$ the curve coincides with that of the analytical results.

\section{Conclusion}

In conclusion, the size of the local quadrature and support domain affect the accuracy and performance of the MLPG methods and it also show a great influence of the choice of field nodes distribution number. The conver-

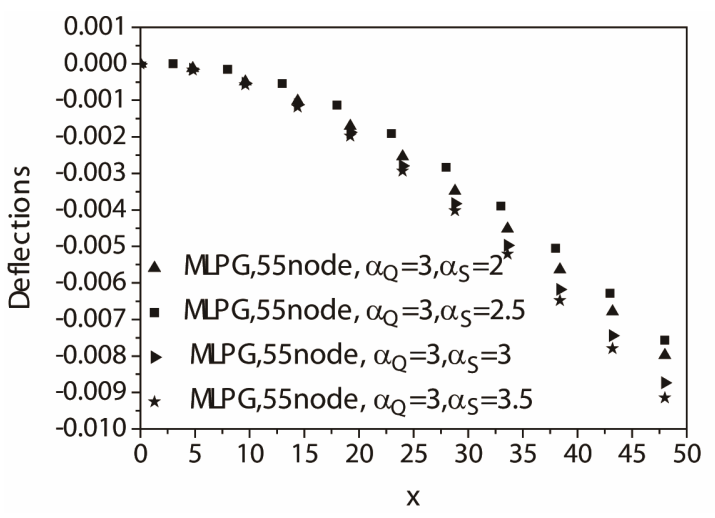

Figure 7. Deflections at the central axis at $y=0$ of the plate for different values of $\alpha_{\mathrm{s}}\left(\alpha_{\mathrm{s}}=2,2.5,3,3.5\right)$.

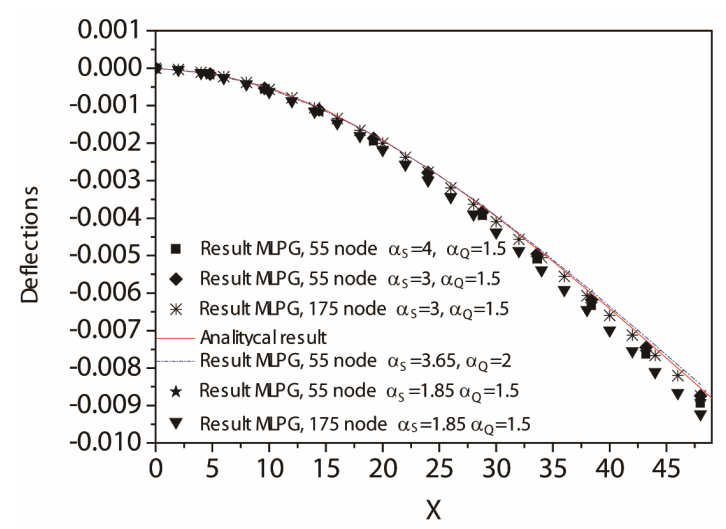

Figure 8. Deflections as a function of $x$ at $y=0$, for $n_{t}=55,175$ and different value of $\alpha_{s}$ and $\alpha_{Q}$. gence and accuracy of MLPG method can still be better by using a number of appropriate nodes in a large domain when the support sizing coefficient $\alpha_{S}$ can be chosen and $\alpha_{Q}$ is fixed. In our numerical examples the MLPG gives a very close value in comparison with the analytical results.

\section{REFERENCES}

[1] T. Belyschko, Y. Y. Lu and L. Gu, "Element-Free Galerkin Methods," International Journal for Numerical Methods, Vol. 37, No. 2, 1994, pp. 229-256. doi:10.1201/9781420082104.ch2

[2] S. N. Atluri and S. Shen, "The Meshless Local PetrovGalerkin (MLPG) Method," Tech Science Press, Forsyth, 2002.

[3] G. R. Liu, "Mesh Free Methods, Moving beyond the Finite Element Method," CRC, Boca Raton, 2003.

[4] S. N. Atluri, "The Meshless Method, (MLPG) for Domain \& BIE Discretizations," Tech Science Press, Forsyth, 2004.

[5] S. N. Atluri, H. T. Liu and Z. D.Han, "Meshless Local Petrov-Galerkin (MLPG) Mixed Collocation Method For Elasticity Problems," Tech Science Press CMES, Vol. 14, No. 3, 2006, pp. 141-152. doi:10.1152/jn.00885.2006

[6] S. N. Atluri1, Z. D. Hanl and A. M. Rajendran, “A New Implementation of the Meshless Finite Volume Method, through the MLPG 'Mixed Approach'," Tech Science Press CMES, Vol. 6, No. 6, 2004, pp. 491-513.

[7] J. Sladek, V. Sladek and C. Zhang, "Application of Meshless Local Petrov-Galerkin (MLPG) Method to Elastodynamic Problems in Continuously Non-Homogeneous Solids," Computer Modeling in Engineering \& Sciences, Vol. 4, No. 6, 2003, pp. 637-648.

[8] S. N. Atluri, J. Y. Cho and H. G. Kim, "Analysis of Thin Beams, Using the Meshless Local Petrov-Galerkin Method, with Generalized Moving Least Squares Interpolations," Computational Mechanics, Vol. 24, No. 5, 1999, pp. 334-347. doi:10.1007/s004660050456

[9] Y. T. Gu and G. R. Liu, "A Meshless Local PetrovGalerkin (MLPG) Formulation for Static and Free Vibration Analyses of Thin Plates," Computer Modeling in Engineering \& Sciences, Vol. 2, No. 4, 2001, pp. 463-476.

[10] J. Sladek, V. Sladek, C. Zhang and M. Schanz, "Meshless Local Petrov-Galerkin Method for Continuously Non-Homogeneous Viscoelastic Solids," Computational Mechanics, Vol. 37, No. 3, 2006, pp. 279-289. doi:10.1007/s00466-005-0715-0

[11] G. R. Liu and Y. T. Gu, "Meshless Local Petrov-Galerkin (MLPG) Method in Combination with Finite Element and Boundary Element Approaches," Computational Mechanics, Vol. 26, No. 6, 2000, pp. 536-546. doi: $10.1007 / \mathrm{s} 004660000203$

[12] S. N. Atluri and T. Zhu, "A New Meshless Local PetrovGalerkin (MLPG) Approachs in Computational Mechanics," Computational Mechanics, Vol. 22, No. 2, 1998, pp. 
117-127. doi:10.1007/s004660050346

[13] T. Belytschko, Y. Krogauz, D. Organ, M. Fleming and P. Krysl, "Meshless Methods: An Overview and Recent Developments," Computer Methods in Applied Mechanics and Engineering, Vol. 139, No. 1, 1996, pp. 3-47. doi:10.1016/S0045-7825(96)01078-X
[14] S. Drapier and R. Fortunée, "Méthodes Numériques d'Approximations et de Résolution en Mécaniques," 2010.

[15] S. P. Timoshenko and J. N. Goodier, "Theory of Elasticity," 3rd Edition, McGraw Hill, New York, 1970. 